\title{
1. Development of a ReaxFF Reactive Force Field for Ettringite and 2 Study of its Mechanical Failure Modes from Reactive Dynamics ${ }_{3}$ Simulations
}

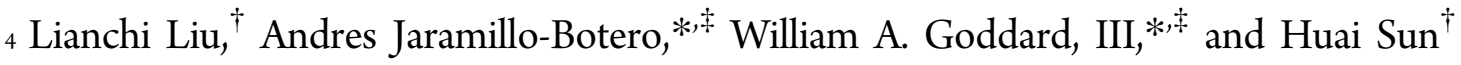 \\ $5{ }^{\dagger}$ Computational Chemistry Group, School of Chemistry and Chemical Engineering, Shanghai Jiao Tong University, 800 Dongchuan \\ 6 Road, Min Hang, Shanghai 200240, China \\ $7{ }^{\ddagger}$ Materials and Process Simulation Center (MC 139-74), Chemistry and Chemical Engineering Division, California Institute of \\ 8 Technology, 1200 East California Boulevard, Pasadena, California 91125, United States
}

10 ABSTRACT: Ettringite is a hexacalcium aluminate trisulfate

11 hydrate mineral that forms during Portland cement hydration.

12 Its presence plays an important role in controlling the setting

13 rate of the highly reactive aluminate phases in cement paste

14 and has also been associated with severe cracking in cured

15 hardened cement. To understand how it forms and how its

16 properties influence those of hardened cement and concrete,

17 we have developed a first-principles-based ReaxFF reactive

18 force field for $\mathrm{Ca} / \mathrm{Al} / \mathrm{H} / \mathrm{O} / \mathrm{S}$. Here, we report on the

19 development of this ReaxFF force field and on its validation

20 and application using reactive molecular dynamics (RMD)

21 simulations to characterize and understand the elastic, plastic,

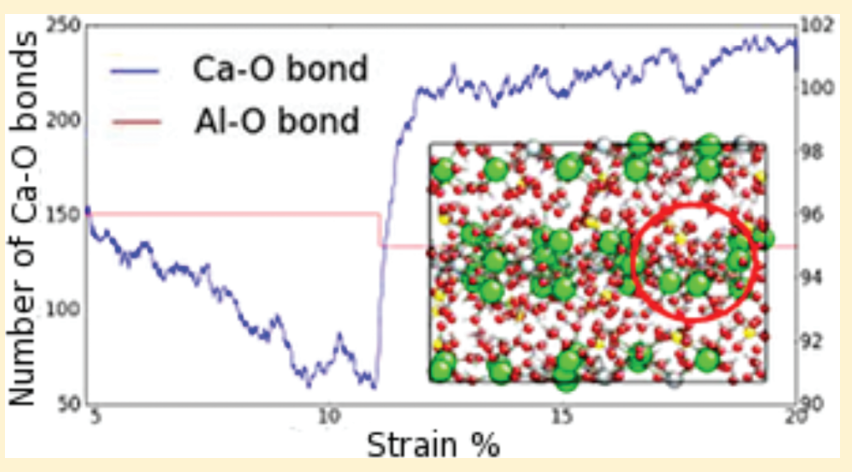
and failure response of ettringite at the atomic scale. The

ReaxFF force field was validated by comparing the lattice parameters, pairwise distribution functions, and elastic constants of an ettringite crystal model obtained from RMD simulations with those from experiments. The predicted results are in close agreement with published experimental data. To characterize the atomistic failure modes of ettringite, we performed stress-strain simulations to find that $\mathrm{Ca}-\mathrm{O}$ bonds are responsible for failure of the calcium sulfate and tricalcium aluminate (C3A) column in ettringite during uniaxial compression and tension and that hydrogen bond re-formation during compression induces an increase in plastic strain beyond the material's stress-strain proportionality limit. These results provide essential insight into understanding the mechanistic role of this mineral in cement and concrete degradation, and the ReaxFF potential developed in this work serves as a fundamental tool to further study the kinetics of hydration in cement and concrete.

\section{INTRODUCTION}

31 The trisulfate mineral ettringite, with chemical formula $32 \mathrm{Ca}_{6}\left[\mathrm{Al}(\mathrm{OH})_{6}\right]_{2}\left(\mathrm{SO}_{4}\right)_{3} 26 \mathrm{H}_{2} \mathrm{O}$, is a precipitate formed in 33 hydrated Portland cement as a result of the reaction of calcium 34 aluminate (A) with calcium sulfate (C), and its presence 35 depends on the ratio $\mathrm{C} 3 \mathrm{~A}$. Changes in this ratio as well as the 36 permeability of ettringite to water and its reactions with other 37 chemical species (e.g., sulfates) affect its structural evolution 38 during cement hydration and, consequently, cement properties. 39 The formation of ettringite during the early cement hydration 40 stage plays an important role in controlling the setting rate of 41 the highly reactive aluminate phases. Its formation results in a 42 volume increase in the fresh, plastic concrete, which has also 43 been associated spatially with severe cracking in cured hardened 44 concrete $^{1}$ during what is referred to as delayed ettringite 45 formation, or DEF, and during cement degradation via sulfate 46 attacks. $^{2}$

47 To help settle the mechanistic issues, it would be most useful 48 to understand the atomistic processes involved with mechan- ically loaded ettringite. However, the quantum mechanics 49 (QM) methods usually applied to studies of chemical reaction 50 processes are not capable of treating the spatial and time scales 51 involved. Consequently, we have used QM to train the 52 parameters of a ReaxFF ${ }^{3}$ reactive force field and then used it 53 to simulate the physical properties and reaction processes that 54 occur during ettringite loading.

This paper focuses on investigating the chemical processes 56 relevant to the mechanical properties of ettringite, including its 57 failure modes under pressure loading, using reactive molecular 58 dynamics (RMD). We first develop the ReaxFF first-principles- 59 based reactive force field to describe the structures, energetics, 60 and forces from QM on prototypical systems. Then, we carried 61 out RMD to study the decomposition and growth of various 62 phases over a broad set of conditions. In section 2.1.1, we 63

Received: October 21, 2011

Revised: February 27, 2012 
64 describe the ReaxFF reactive force field developed (see 65 Supporting Information), and section 3.1 provides a description 66 of several validation tests performed on ettringite. Then, in 67 section 3.2, we describe how ReaxFF was used to characterize 68 the mechanical response and failure modes of ettringite under 69 strain loading. We consider that these modes of failure are 70 critical to understand and control the long-term properties of 71 Portland-based concrete structures.

\section{COMPUTATIONAL DETAILS}

72 Modern QM methods provide reaction surfaces and geometries 73 of molecular systems sufficiently accurate to predict a reaction 74 mechanism in advance of experiment. However, scaling of 75 computational cost with system size (i.e., the number of atoms) 76 limits these methods to within $\sim 100$ atoms. QM can be used to 77 derive the forces for describing the dynamical equations of 78 motion of all particles involved; however, again, computational 79 costs restrict the time scale to a few picoseconds. This stems 80 from the high cost of solving self-consistently the Schrödinger 81 equation for all nuclear and electronic interactions. Con82 sequently, the use of QM to study the reaction processes 83 occurring in ettringite as the system is dynamically strain 84 loaded/unloaded is well beyond the current practical limits of 85 QM calculations.

86 2.1. ReaxFF Force Field. To provide a practical solution to 87 such problems, we developed the ReaxFF reactive force field, ${ }^{3}$ 88 which provides nearly the accuracy of the best QM but at a 89 computational cost of solving Newton's equation of motion on 90 nuclei only. This allows us to accurately compute the atomic 91 interactions found in ettringite. Essential elements of ReaxFF 92 include:

93 1. an analytic relation between bond order and bond 94 distance and between bond energy and bond order that 95 establishes smooth transitions as bonds form and break

96 2. a rule for how charge depends on geometry and atomic 97 character that allows charges to flow continuously as 98 bonds form and break

99 3. functional forms that describe well the barrier heights of 100 both allowed and forbidden reactions, including the 101 effects of resonance.

102 ReaxFF partitions the total energy of interactions similarly to 103 those found in nonreactive force fields like Dreiding, that is, in 104 valence, nonbonded, and H-bond terms, but it introduces bond 105 order dependencies on valence terms and additional potential 106 energy corrections to properly describe bond formation and 107 dissociation under different environmental conditions. The 108 overall energy of the system in ReaxFF is given as

$$
\begin{aligned}
U_{\text {Total }}= & E_{\text {bonds }}+E_{\text {angles }}+E_{\text {torsions }}+E_{\text {H-bonds }} \\
& +E_{\mathrm{vdW}}+E_{\text {Coulomb }}+E_{\mathrm{lp}}+E_{\text {over }}+E_{\text {under }} \\
& +E_{\text {pen }}+E_{\text {coa }}+E_{C_{2}}+E_{\text {triple }}+E_{\text {conj }}
\end{aligned}
$$

109 where $E_{\mathrm{lp}}$ corresponds to the lone pair energy penalty based on 110 the number of lone pairs around an atom, $E_{\text {over }}$ to the energy 111 penalty for overcoordinated atoms, $E_{\text {under }}$ to the energy 112 contribution for the resonance of the $\pi$-electron between 113 attached undercoordinated atomic centers, $E_{\text {pen }}$ to an energy 114 penalty needed to reproduce the stability of systems with 115 double bonds sharing an atom in a valency angle (e.g., allene), $116 E_{\text {coa }}$ to the three-body conjugation term to describe the stability 117 of $-\mathrm{NO}_{2}$ groups, $E_{\mathrm{C}_{2}}$ to an energy contribution that captures the stability of $\mathrm{C}_{2}, E_{\text {triple }}$ to a triple bond stabilization energy in 118 $\mathrm{CO}$ bonded pairs, and $E_{\text {conj }}$ to the contribution of conjugation 119 effects to the molecular energy.

In ReaxFF, each element is described using a single atom 121 type, even through bonding during a chemical reaction. This 122 also facilitates the transferability of the parameters. For each 123 element, several parameters to describe valence bond 124 parameters, electronegativity, hardness, and other effects are 125 optimized to reproduce the QM-derived energies and charges. 126 ReaxFF can reproduce energies near the accuracy of the 127 training set at a much lower computational cost. The reactive 128 site or pathways need not be predefined in a ReaxFF MD 129 simulation, and connectivities between the atoms are not 130 predetermined. Instead, bond orders are calculated from the 131 interatomic distances $r_{i j}$. By using a bond length/bond order 132 relationship, smooth transitions from nonbonded to single, 133 double, and triple bonded systems, and vice versa, are obtained. 134 This requires bond orders to be updated every iteration, for 135 single $(\sigma)$, double $(\pi)$, and triple $(\pi-\pi)$ bonds as a function of 136 interatomic distances $\left(r_{i j}\right)$, using scaling parameters $p_{\mathrm{BO}, 1-6} 137$ fitted from QM calculations on small representative systems 138 and making all of the connectivity-dependent interactions 139 bond-order-dependent to ensure that their energy contribu- 140 tions disappear upon bond dissociation. Bond orders are 141 computed initially as

$$
\begin{aligned}
\mathrm{BO}_{i j}^{\prime}= & \exp \left[p_{\mathrm{BO}, 1}\left(\frac{r_{i j}}{r_{0}^{\sigma}}\right)^{p_{\mathrm{BO}, 2}}\right]+\exp \left[p_{\mathrm{BO}, 3}\left(\frac{r_{i j}}{r_{0}^{\pi}}\right)^{p_{\mathrm{BO}, 4}}\right] \\
& +\exp \left[p_{\mathrm{BO}, 5}\left(\frac{r_{i j}}{r_{0}^{\pi-\pi}}\right)^{p_{\mathrm{BO}, 6}}\right]
\end{aligned}
$$

From these (uncorrected) bond orders, $\mathrm{BO}^{\prime}$, an uncorrected 143 atomic overcoordination $\Delta^{\prime}$ is calculated as the difference 144 between the total bond order around the atom and the number 145 of its bonding electrons, $\mathrm{Val}$, that is

$$
\Delta_{i}^{\prime}=-\mathrm{Val}_{i}+\sum_{j=1}^{\text {neighbors }(i)} \mathrm{BO}_{i j}^{\prime}
$$

Equation 3 is then used to obtain corrected bond orders, $\mathrm{BO}_{i j}$, 147 and subsequently a new corrected overcoordination. For more 148 details on the exact functional forms, see the Supporting 149 Information from ref 4.

The bond energies are then computed from the corrected 151 bond orders by

$$
\begin{aligned}
E_{\text {bond }}= & -D_{\mathrm{e}}^{\sigma} \mathrm{BO}_{i j}^{\sigma} \exp \left[p_{\text {be1 }}\left(1-\left(\mathrm{BO}_{i j}^{\sigma}\right)^{\left.p_{\mathrm{be} 2}\right)}\right]-D_{\mathrm{e}}^{\pi} \mathrm{BO}_{i j}^{\pi}\right. \\
& -D_{\mathrm{e}}^{\pi-\pi} \mathrm{BO}_{i j}^{\pi-\pi}
\end{aligned}
$$

where $D_{\mathrm{e}}$ is the corresponding dissociation energy for each 153 bond type and $p_{\text {be1,2 }}$ correspond to atomic parameters that are 154 fitted from QM calculations on small representative cases. 155 According to eq 4, the bond energy term $\mathrm{E}_{\text {bond }}$ gradually 156 disappears as the bond order $\mathrm{BO}$ approaches zero during the 157 dissociation of a bond. The $i j$ valence angle and torsion angle 158 energy terms are also functions of bond orders and go to zero 159 upon bond dissociation. This ensures continuity of the energy 160 and forces during bond dissociation/formation. 
Table 1. Atom Parameters

\begin{tabular}{|c|c|c|c|c|c|c|c|c|}
\hline \multirow[t]{2}{*}{ atom type } & \multicolumn{3}{|c|}{ EEM parameters } & \multicolumn{5}{|c|}{ bond order correction } \\
\hline & $\chi(\mathrm{eV})$ & $\eta(\mathrm{eV})$ & $\gamma(\AA ̊)$ & $p_{\text {ov/un }}$ & $p_{\text {val3 }}$ & $p_{\text {boc4 }}$ & $p_{\text {boc3 }}$ & $p_{\text {bocs }}$ \\
\hline $\mathrm{H}$ & 3.7248 & 9.6093 & 0.8203 & -19.4571 & 4.2733 & 3.0408 & 2.4197 & 0.0003 \\
\hline $\mathrm{O}$ & 8.5000 & 8.3122 & 1.0898 & -3.5500 & 2.9000 & 3.5027 & 0.7640 & 0.0021 \\
\hline $\mathrm{Ca}$ & -1.7130 & 6.5096 & 1.0000 & -2.0000 & 3.8301 & 49.9248 & 0.3370 & 0.0000 \\
\hline $\mathrm{Al}$ & 1.7429 & 6.8319 & 0.4265 & -22.7101 & 1.7045 & 20.0000 & 0.2500 & 0.0000 \\
\hline$S$ & 5.8284 & 8.2545 & 1.0336 & -11.0000 & 2.7466 & 5.7487 & 23.2859 & 12.7147 \\
\hline
\end{tabular}

Table 2. Bond Energy and Bond Order Parameters $\left(D_{\mathrm{e}}\right.$ in $\mathrm{kcal} / \mathrm{mol} ; \boldsymbol{r}$ in $\AA$ )

\begin{tabular}{ccrrrrrrrrr} 
atom pair & $D_{\mathrm{e}, \sigma}$ & $D_{\mathrm{e}, \pi}$ & \multicolumn{1}{c}{$p_{\text {be1 }}$} & $p_{\text {be2 }}$ & $r_{\sigma}$ & $p_{\text {bo1 }}$ & $p_{\text {bo2 }}$ & $r_{\pi}$ & $p_{\text {bo3 }}$ & $p_{\text {bo4 }}$ \\
$\mathrm{Al}-\mathrm{O}$ & 227.9327 & 0.0 & -0.8375 & 0.3686 & 1.5382 & -0.1740 & 5.2057 & & \\
$\mathrm{~S}-\mathrm{O}$ & 161.0562 & 220.0 & 0.6070 & 0.7150 & 1.4608 & -0.1109 & 5.4501 & 1.3987 & -0.2741 & 8.3216 \\
\hline
\end{tabular}

162 Nonbonded interactions (van der Waals, Coulomb) are 163 calculated between every atom pair, irrespective of connectivity 164 and avoiding excessive close-range contributions by shielding. 165 ReaxFF parameters have been developed for a variety of 166 materials, including hydrocarbons, ${ }^{3}$ silicon/silicon oxide, ${ }^{5}$ 167 metals, ${ }^{6}$ metal oxides, ${ }^{4}$ and metal hydrides, ${ }^{7}$ among others. It 168 has also been used to study a variety of complex chemical 169 systems. $^{4,8-10}$

170 2.1.1. ReaxFF Force Field for Ettringite. We developed the 171 ettringite ReaxFF force field to accurately reproduce the first172 principles $\mathrm{QM}$ interactions in hydrous calcium aluminum 173 sulfate systems $(\mathrm{H} / \mathrm{Ca} / \mathrm{Al} / \mathrm{S} / \mathrm{O})$ and to provide a way to 174 extend the first-principles accuracy to the much larger length 175 and time scales needed to examine the processes of interest 176 here.

177 We started with recently derived ReaxFF parameters for $\mathrm{Al} /$ $178 \mathrm{O}$ from ref 11 and $\mathrm{Ca} / \mathrm{O}$ from ref 12 . These parameters had 179 been optimized to describe the crystals of various phases of $\mathrm{Al}$ 180 and $\mathrm{Ca}$ and of various aluminum oxides and calcium oxides. As 181 discussed below, we prepared additional training data to 182 complete the $\mathrm{Al}, \mathrm{Ca}, \mathrm{O}, \mathrm{S}$, and $\mathrm{H}$ interactions present in 183 ettringite and validated ReaxFF charges for its constituent 184 molecular motifs against QM Mulliken populations.

185 This involved performing QM density functional theory 186 (DFT) calculations on representative finite clusters using the 187 B3LYP hybrid functional with the LACVP** basis set using the 188 Jaguar 4.2 package. ${ }^{13}$ The calculat 0 on periodic systems used 189 the PBE functional with double-5 plus polarization basis of 190 contracted Gaussian functions with SeqQuest. ${ }^{14}$ The complete 191 QM-derived force field training set includes EOS, atomic bond 192 energy curves, dissociation energies, angle bend energy curves, 193 and other relevant material properties, such as heats of 194 formation, overcoordination, and others, for all possible 195 interactions between $\mathrm{Ca}, \mathrm{Al}, \mathrm{O}, \mathrm{S}, \mathrm{H}$, and $\mathrm{H}_{2} \mathrm{O}$. Angle bend 196 energies in ettringite were also trained to achieve an accurate 197 geometrical representation of the crystal structure. These 198 included $\mathrm{Ca}-\mathrm{Ca}-\mathrm{Ca}$ in a triangle $\left[\mathrm{Ca}(\mathrm{OH})_{2}\right]_{3}$ bend to 199 represent the potential surface of $\mathrm{Ca}-\mathrm{O}-\mathrm{Ca}$ and $\mathrm{O}-\mathrm{Ca}-\mathrm{O}$ 200 angles, the distance of $\mathrm{Al}-\mathrm{Al}$ in $\mathrm{Ca}\left[\mathrm{AlO}_{4}\right]_{2}$ scanned to 201 represent the potential surface of the $\mathrm{Ca}-\mathrm{O}-\mathrm{Al}$ angle, which 202 is typical in ettringite along the c direction, the $\mathrm{Al}-\mathrm{O}-\mathrm{S}$ angle 203 in $\mathrm{Al}(\mathrm{OH})_{2}-\mathrm{O}-\mathrm{SO}_{3} \mathrm{H}$, and the $\mathrm{Ca}-\mathrm{O}-\mathrm{S}$ angle in $\mathrm{CaSO}_{4}$ gas.

\section{RESULTS AND DISCUSSION}

204 The geometries and energies of the species and complexes in 205 ettringite were calculated using DFT, and the ReaxFF force 206 field parameters were optimized to minimize the differences between the DFT and ReaxFF energies. Tables $1-5$ provide a $207 \mathrm{t} 1 \mathrm{t} 2 \mathrm{t} 3 \mathrm{t} 4 \mathrm{t} 5$ summary of the ReaxFF force field parameters for ettringite. 208

Table 3. van der Waals Parameters

\begin{tabular}{ccccc} 
atom pair & $R_{\mathrm{vdW}}(\AA)$ & $\varepsilon(\mathrm{kcal} / \mathrm{mol})$ & $\alpha$ & $\gamma_{\mathrm{vdW}}$ \\
$\mathrm{Al}-\mathrm{O}$ & 1.8928 & 0.1745 & 11.2476 & 7.3490 \\
$\mathrm{~S}-\mathrm{O}$ & 1.7931 & 0.2047 & 10.2391 & 8.2411 \\
\hline
\end{tabular}

The corresponding ReaxFF potential functional forms may be 209 consulted in ref 4 . These optimized parameters are available in 210 a ffield file (see the Supporting Information sectionof ref 4) 211 that can be directly used in the freely available "Large-scale 212 Atomic/Molecular Massively Parallel Simulator” (LAMMPS ${ }^{15}$ ) 213 molecular dynamics program from Sandia National Laborato- 214 ries.

215

3.1. Ettringite ReaxFF Force Field Validation. We began 216 by constructing a $2 \times 2 \times 1$ molecular triclinic unit supercell 217 model from the crystal structure determined by Moore, ${ }^{16}$ as 218 shown in Figure 1. This molecular model served as the basic $219 \mathrm{fl}$ structure for the calculations reported here.

220

Charges play an important role in computing charge 221 distributions and overall electrostatics during bond breaking 222 and formation in ReaxFF. To confirm the accuracy of the net 223 molecular charges calculated with ReaxFF (using the charge 224 equilibration method QEq from Rappe et $\mathrm{al}^{17}$ ) on subspecies 225 found in ettringite, including $\mathrm{H}_{2} \mathrm{SO}_{4}, \mathrm{CaSO}_{4}, \mathrm{Al}(\mathrm{OH})_{3}, 226$ $\mathrm{Al}(\mathrm{OH})_{2}-\mathrm{O}-\mathrm{SO}_{4} \mathrm{H}$, and the $\mathrm{C}_{3} \mathrm{~A}_{2}$ cluster, we compared 227 these to Mulliken charge populations. Figure 2 shows that $228 \mathrm{f} 2$ ReaxFF charges for the different species of interest are in close 229 agreement with the QM results.

230

In addition to validating bond energies, EOS, and other 231 properties, we compared DFT-calculated angle bend energies 232 against equivalent scans using the ettringite ReaxFF force field. 233 Angle bend energies for $\mathrm{Ca}-\mathrm{O}-\mathrm{Ca}, \mathrm{O}-\mathrm{Ca}-\mathrm{O}, \mathrm{Ca}-\mathrm{O}-\mathrm{Al}, 234$ $\mathrm{Al}-\mathrm{O}-\mathrm{S}$, and $\mathrm{Ca}-\mathrm{O}-\mathrm{S}$ angles are important to the structural 235 conformity of the model (see Figure 3).

$236 \mathrm{f} 3$

To determine the structural stability and conformity of our 237 model during room-temperature dynamics, the $2 \times 2 \times 1238$ ettringite supercell model shown in Figure 1 was equilibrated 239 for 20 ps in an NPT ensemble with RMD calculations at $300 \mathrm{~K} 240$ and a 0.25 fs time step. The last 10 ps were used for statistical 241 calculation of the cell parameters and for computing radial 242 distribution functions (RDFs). The calculated unit cell 243 parameters accurately reproduce the experimental values within 244 $\sim 4 \%$, as shown in Table 6. The total averaged RDFs and all of 245 t6 the RDF pairs were also calculated (Figure 4), revealing $246 \mathrm{ft}_{4}$ 
Table 4. Angle Parameters

\begin{tabular}{|c|c|c|c|c|c|}
\hline \multicolumn{2}{|l|}{ angle type } & $\Theta_{0,0}(\operatorname{deg})$ & \multicolumn{3}{|c|}{$p_{\text {val1 }}(\mathrm{kcal} / \mathrm{mol})$} \\
\hline $\mathrm{O}-\mathrm{Al}-\mathrm{O}$ & & 54.0864 & \multicolumn{3}{|c|}{9.7594} \\
\hline $\mathrm{O}-\mathrm{O}-\mathrm{Al}$ & & 34.4326 & \multicolumn{3}{|c|}{25.9544} \\
\hline $\mathrm{H}-\mathrm{O}-\mathrm{Al}$ & & 90.0000 & \multicolumn{3}{|c|}{19.7491} \\
\hline $\mathrm{Al}-\mathrm{O}-\mathrm{Al}$ & & 23.7270 & \multicolumn{3}{|c|}{19.5973} \\
\hline $\mathrm{Ca}-\mathrm{O}-\mathrm{Al}$ & & 79.6174 & \multicolumn{3}{|c|}{23.7025} \\
\hline $\mathrm{Al}-\mathrm{O}-\mathrm{S}$ & & 102.1915 & \multicolumn{3}{|c|}{7.3381} \\
\hline $\mathrm{Ca}-\mathrm{O}-\mathrm{S}$ & & 114.2370 & \multicolumn{3}{|c|}{19.7117} \\
\hline $\mathrm{O}-\mathrm{S}-\mathrm{O}$ & & 81.7931 & \multicolumn{3}{|c|}{31.2043} \\
\hline $\mathrm{O}-\mathrm{O}-\mathrm{S}$ & & 83.9753 & \multicolumn{3}{|c|}{31.0715} \\
\hline & & & & & \\
\hline sion angle & $V_{1}$ & $V_{2}$ & $V_{3}$ & $p_{\text {torl }}$ & $p_{\text {cot1 }}$ \\
\hline$-\mathrm{O}-\mathrm{S}-\mathrm{O}$ & 2.5000 & 2.5000 & 0.2237 & -10.0000 & -1.0000 \\
\hline -O-S-X & 0.1000 & 50.0000 & 0.0100 & -10.0000 & 0.0000 \\
\hline
\end{tabular}

247 predicted structures from our MD simulations that are in close 248 agreements with experiments.

249 3.2. Elastic Constants of Ettringite. To characterize the 250 stiffness of ettringite, we calculated its elastic constants from the 251 linear relation between the stress and strain tensors in the limit 252 of infinitesimal deformation. We computed the adiabatic 253 constants (at $0 \mathrm{~K}$ ) considering that the thermal expansion of 254 the unit cell at ambient temperature, relative to that at $0 \mathrm{~K}$, was 255 within the error bars of our method (as estimated from our 256 NPT-RMD structure equilibration simulations at $300 \mathrm{~K}$ ). This 257 involved deforming the unit cell by less than $1 \%$ in the different 258 directions of interest and calculating the change in the 259 corresponding stress tensor components. This way, we avoided 260 the higher uncertainty and computational costs of calculating 261 the elastic constants at ambient temperature, which requires 262 time averages of differential properties (e.g., stress tensor 263 components using a canonical or isothermal-isobaric ensemble 264 approach) and balancing the systematic and statistical errors to 265 fully equilibrate the deformed cell before sampling the stress 266 tensor. Table 7 shows that the results from our simulations are

$\begin{array}{cc}p_{\text {val2 }} & p_{\text {coal }} \\ .9476 & 0.0 \\ .1239 & 0.0 \\ .8227 & 0.0 \\ 4.0000 & 0.0 \\ .7958 & 0.0 \\ 0.4786 & 0.0 \\ 0.5240 & 0.0 \\ 6.5492 & -4.4116 \\ .5590 & 0.0\end{array}$

quantitatively consistent with the isothermal elastic constants 267 reported from experiments. ${ }^{18}$

$p_{\text {val7 }}$
3.0000
2.7500
1.0000
0.6619
0.9119
0.5846
3.0000
2.6067
0.8161

$\begin{array}{cc}p_{\text {pen1 }} & p_{\text {val } 4} \\ 0.0 & 1.4400 \\ 0.0 & 1.7141 \\ 0.0 & 2.5337 \\ 0.0 & 1.9380 \\ 0.0 & 1.6244 \\ 0.0 & 1.4022 \\ 0.0 & 0.8335 \\ 0.0 & 3.0000 \\ 0.0 & 1.1776\end{array}$

3.3. Atomistic Failure Modes of Ettringite under 269 Mechanical Loading. To understand the failure modes of 270 ettringite, we carried out RMD deformation simulations, with 271 the ReaxFF force field reported here, along the $y$ and $z 272$ directions on the model system depicted in Figure 1. We 273 started from a relaxed volume $2 \times 2 \times 1$ supercell using NPT- 274 $\mathrm{RMD}$ at $300 \mathrm{~K}$ temperature and $1 \mathrm{~atm}$ of pressure. The system 275 was equilibrated at every $1 \%$ strain for 20 ps up to $15 \%$ strain in 276 the $y$ direction and 20\% strain in the $z$ direction, and the last 10277 ps was used for data collection at each deformation strain. 278

The uniaxial stress-strain characteristics along the $z 279$ direction, under compression and tension, are presented in 280 the Figure 5. These results indicate that the true elastic limit $281 \mathrm{fs}$ along the $z$ direction is at $\sim 3 \%$ under strain in compression and 282 $\sim 7 \%$ in tension, while failure occurs between 11 and $12 \% 283$ deformation. These results also indicate that in this particular 284 direction, ettringite is more resistant under tensile strain than 285 under compression. We observed hydrogen bonds breaking and 286 re-forming during compression, which accounts for the increase 287 in plastic strain in this mode. The longitudinal elastic 288 anisotropy due to the crystallographic structure is $C_{11} / C_{33}=289$ 0.63, while the shear anisotropy is $C_{66} / C_{44}=0.6$. The stiff 290 octahedra chains of $\left[\mathrm{Al}(\mathrm{OH})_{6}\right]_{3}$ - alternating with triplets of 291 $\mathrm{Ca}_{2}^{+}$in eight-fold coordination along the $z$-axis lead to higher 292 stiffness in this direction.

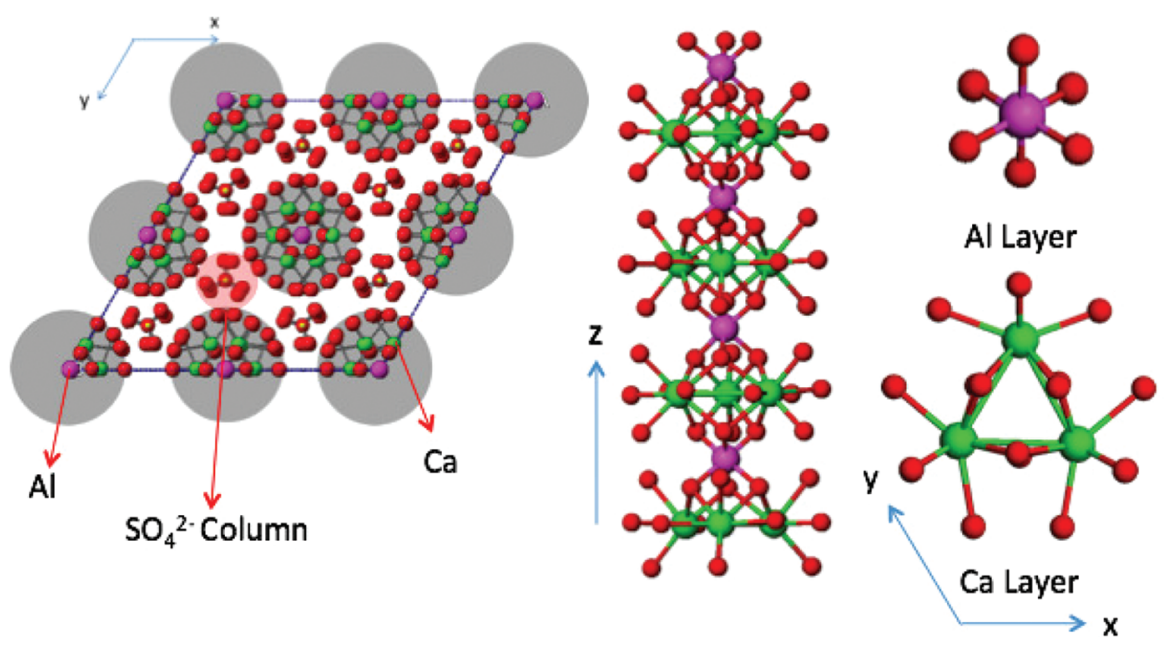

Figure 1. The triclinic $2 \times 2 \times 1$ molecular supercell crystal model of ettringite contains a total of 496 atoms, ignoring hydrogens, arranged in 4 stacked layers (center image) of intercalated $\mathrm{Ca}$ and $\mathrm{Al}$ sublayers (right image). The left image shows the projection of crystal structure on to the (0001) plane. C3A columns lay parallel to the $z$-axis $(c)$ with sulfate and water molecules in the intercolumn channels. The $\mathrm{SO}_{4}^{2-}$ groups are located at the interstitials between columns, along with water molecules, and 132 hydrogen atoms (not shown) on the surface of the columns and around the $\mathrm{SO}_{4}^{2-}$ groups neutralize the system. 

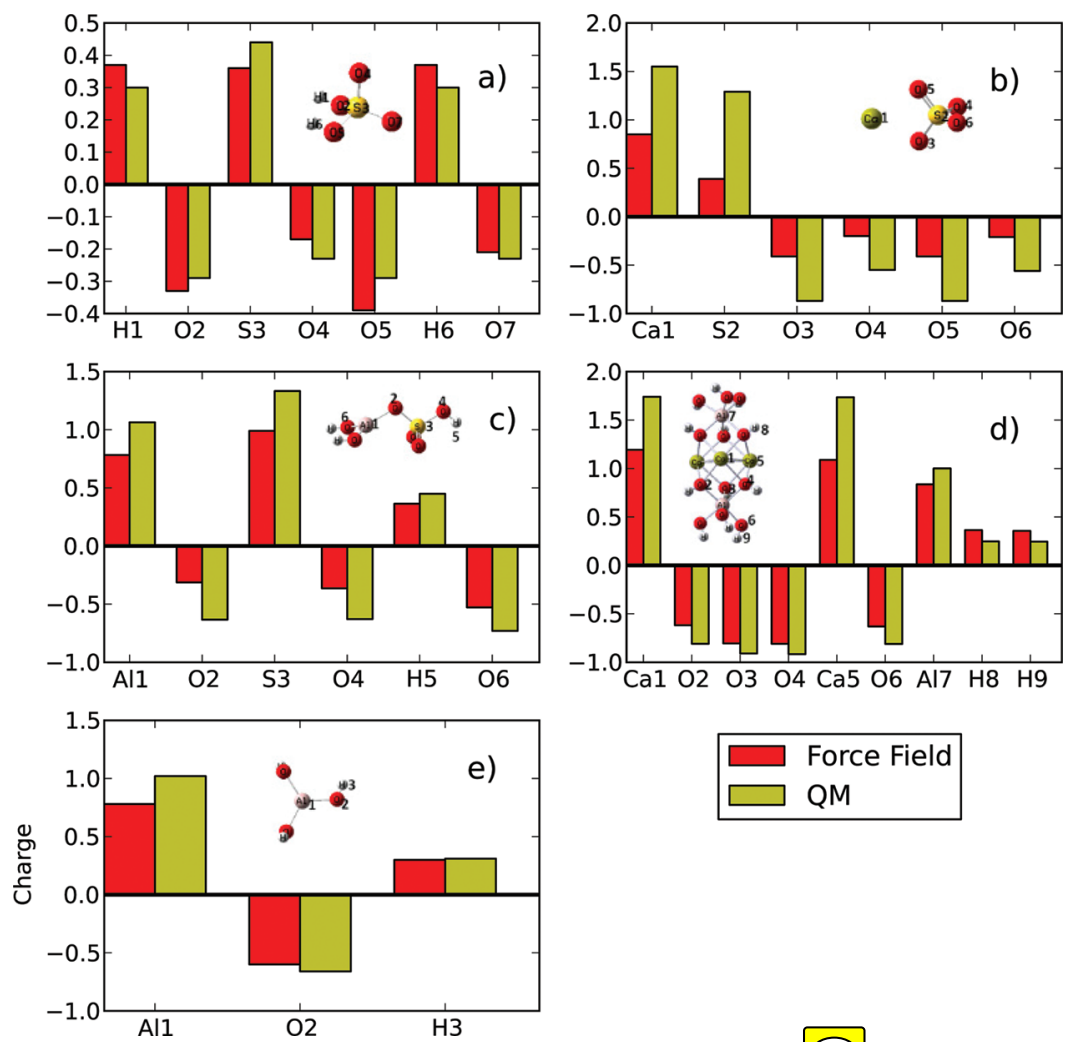

Figure 2. Comparison of the charges for Eettringite from the ReaxFF force field and from QM r $\mathrm{Q}_{\text {en }}$ populations for $(\mathrm{a}) \mathrm{H}_{2} \mathrm{SO}_{4},(\mathrm{~b}) \mathrm{CaSO}_{4},(\mathrm{c})$ the $\mathrm{C}_{3} \mathrm{~A}_{2}$ cluster, (d) $\mathrm{Al}(\mathrm{OH})_{2}-\mathrm{O}-\mathrm{SO}_{4} \mathrm{H}$, and (e) $\mathrm{Al}(\mathrm{OH})_{3}$.
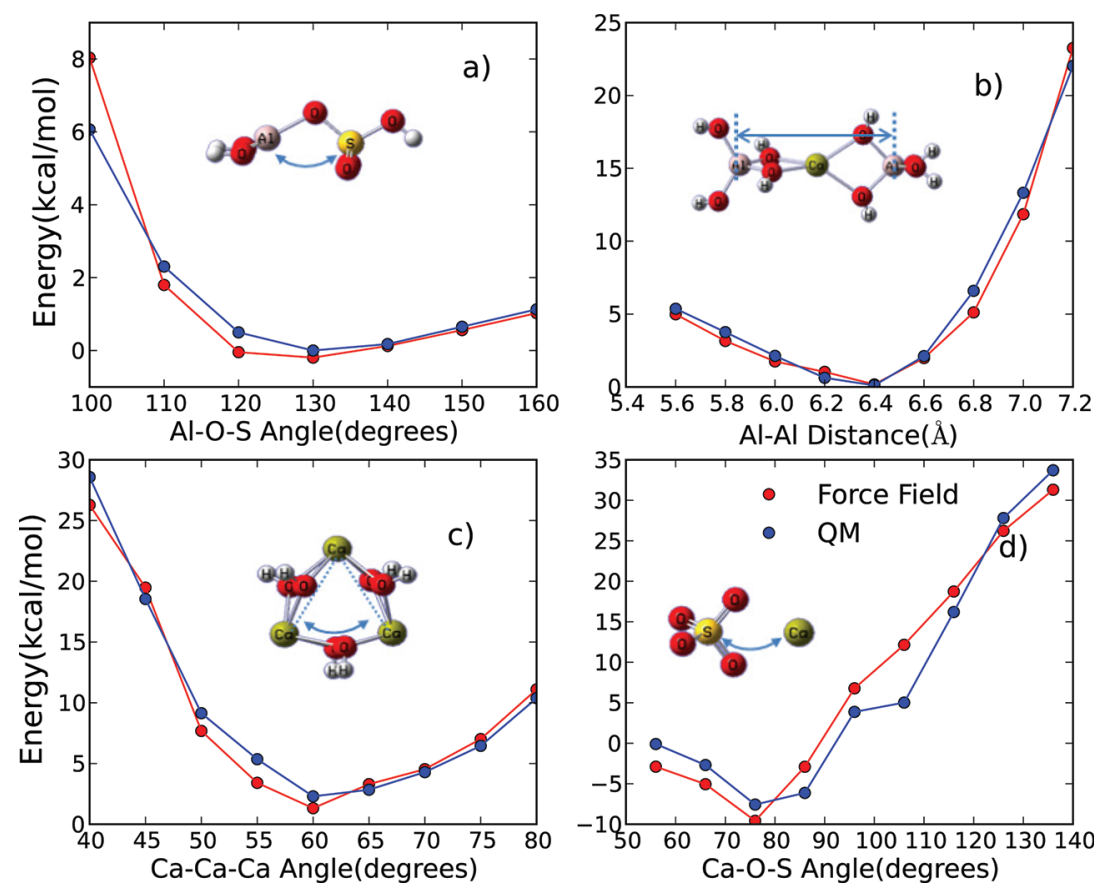

Figure 3. Comparison of the ettringite angle bend energies from ReaxFF and from $\mathrm{QM}$ angle scans for (a) $\mathrm{Ca}-\mathrm{O}-\mathrm{Ca}$ and $\mathrm{O}-\mathrm{Ca}-\mathrm{O}$, (b) $\mathrm{Ca}-\mathrm{O}-$ $\mathrm{Al}$, (c) $\mathrm{Al}-\mathrm{O}-\mathrm{S}$, and (d) $\mathrm{Ca}-\mathrm{O}-\mathrm{S}$ angles.

The stress-strain characteristics of the system along the $y$ 295 direction are shown in Figure 6. Material failure during ${ }_{296}$ compression in the $y$ direction occurs at $\sim 13 \%$ strain and at ${ }_{297} \sim 8 \%$ strain during tension. The intermolecular hydrogen 298 bonding network that provides mechanical stability to the
C3A columnar conformation are responsible for the decreased 299 loading capacity in the $a / b$ directions of the material. 300

The number of bond changes during the dynamics of 301 mechanical strain was used to provide further insight into the 302 failure mechanisms of ettringite. 
Table 6. Comparison between Calculated Ettringite Cell Parameters and Experiment

\begin{tabular}{ccc} 
cell parameters & experimental & ReaxFF NPT-RMD \\
$a / b$ & 11.167 & 11.53 \\
$c$ & 21.360 & 22.22 \\
\hline
\end{tabular}
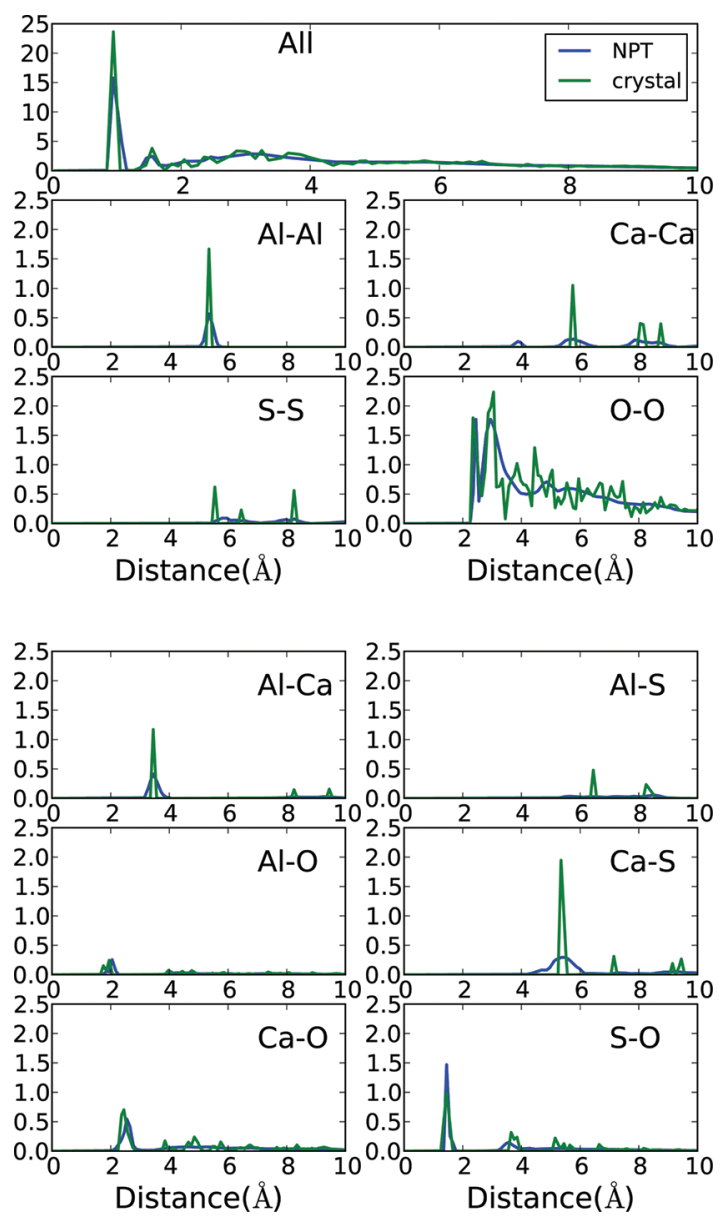

Figure 4. Radial distribution functions (RDFs) for Ettringite; experimental and ReaxFF NPT-RMD simulation results agree.

Figure 7 shows that during the initial stages of compression 305 along the $z$ direction, the number of $\mathrm{Ca}-\mathrm{O}$ bonds increases 306 slowly until $\sim 7 \%$ compression, and they increase at a faster 307 pace thereafter, until the failure point $(\sim 11 \%$ strain $)$. On the

Table 7. Computed (Adiabatic) versus Experimental (Isothermal) Elastic Constants in Ettringite Crystal ${ }^{a}$

\begin{tabular}{lcc}
\hline elastic constants & expt $^{18}$ & ReaxFF \\
$C_{11}$ & $35.1 \pm 0.1$ & 45.4 \\
$C_{12}$ & $21.9 \pm 0.1$ & 25.0 \\
$C_{13}$ & $20.0 \pm 0.5$ & 23.0 \\
$C_{14}$ & $0.6 \pm 0.2$ & 1.9 \\
$C_{33}$ & $55 \pm 1$ & 53.9 \\
$C_{44}$ & $11.0 \pm 0.2$ & 11.1 \\
$C_{66}$ & $6.6^{*} \pm 0.1$ & 8.1 \\
bulk modulus $(K)$ & $27.3 \pm 0.9$ & 26.76 \\
shear modulus $(G)$ & $9.5 \pm 0.8$ & 12.44 \\
Poisson ratio $(\mathrm{v})$ & $0.34 \pm 0.02$ & 0.30
\end{tabular}

${ }^{a}$ Note: $C_{66} *$ is calculated as $\left(C_{11}-C_{12}\right) / 2$.

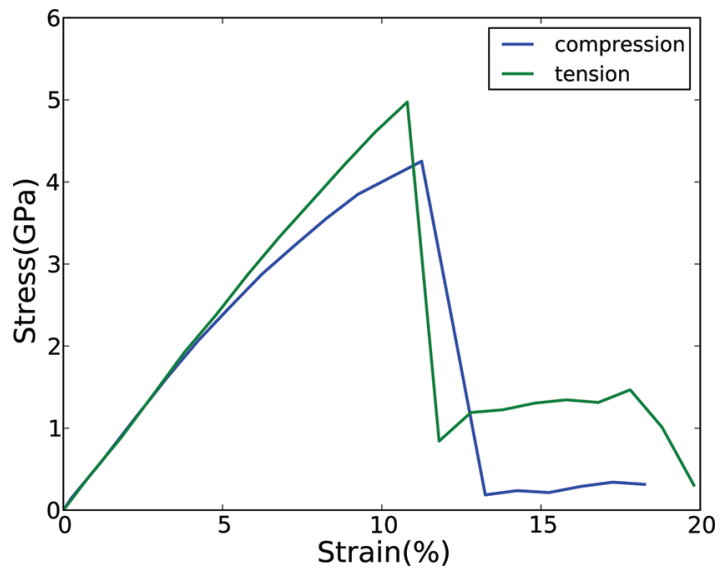

Figure 5. Stress-strain curve along the $z$ direction under compression and tension deformation.

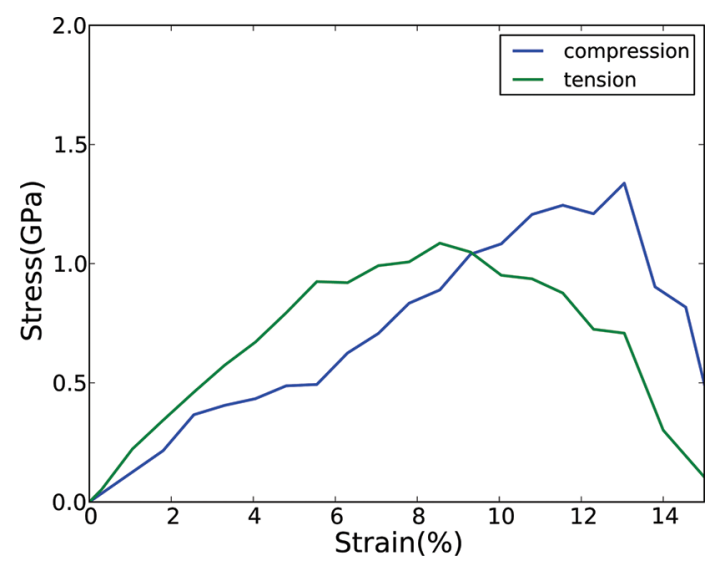

Figure 6. Stress-strain curve along the $y$ triclinic $(a / b)$ direction under compression (blue) and tension (black).

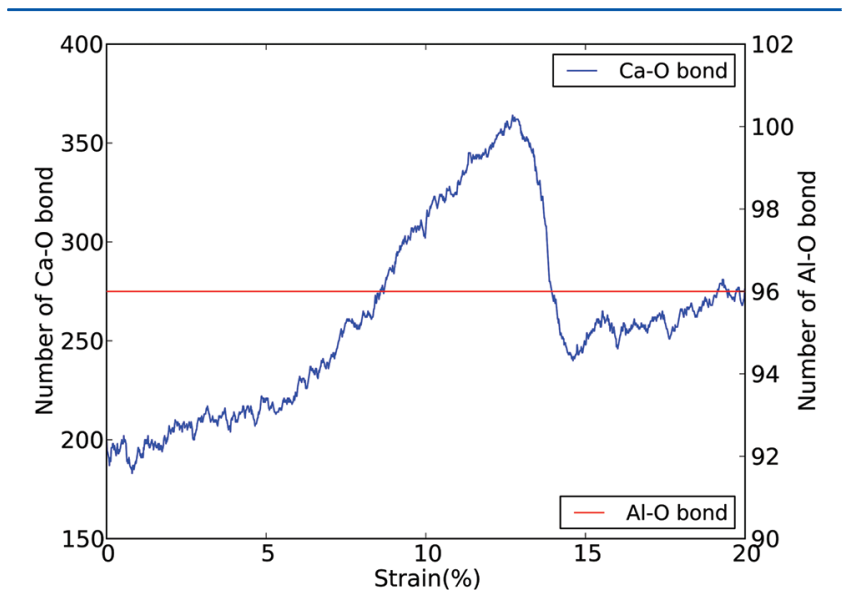

Figure 7. The number of $\mathrm{Ca}-\mathrm{O}$ bonds increases piecewise linearly between 0 and $7 \%$ and 7 and $11 \%$, until failure at $\sim 11 \%$ elongation in the $z$ direction, while the number of $\mathrm{Al}-\mathrm{O}$ bonds remains constant during the full range of compression deformation in the same direction.

other hand, the numbers of $\mathrm{Al}-\mathrm{O}$ bonds do not change during 308 compression; therefore, the failure in this mode is attributed to 309 $\mathrm{Ca}-\mathrm{O}$ bond breaking in the $\mathrm{C} 3 \mathrm{~A}$ column. We observe that 310 during tension, the number of $\mathrm{Ca}-\mathrm{O}$ bonds decreases at a 311 constant rate before reaching the failure point of the material, at 312 $\sim 11 \%$ tension, and sharply increases thereafter (Figure 8). The $313 \mathrm{f} 8$ 


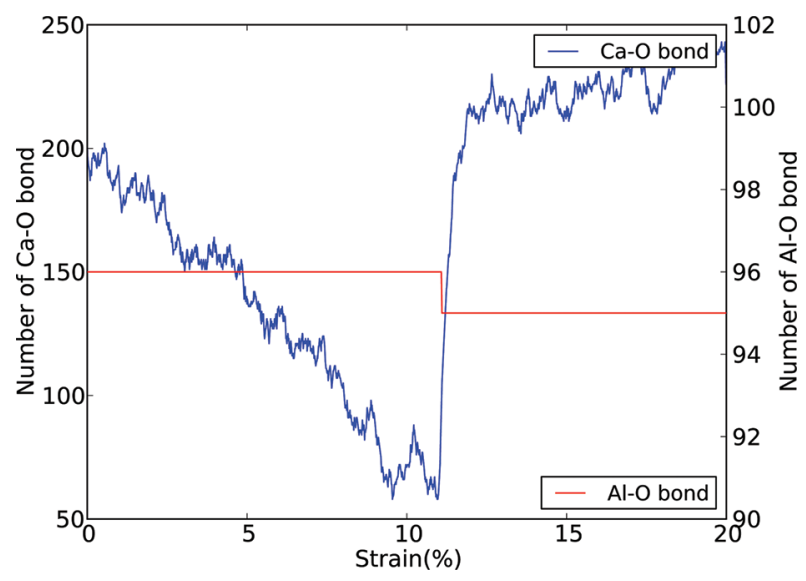

Figure 8. The number of $\mathrm{Ca}-\mathrm{O}$ bonds decreases linearly during elongation in the $z$ direction until failure at $\sim 11 \%$, while the number of $\mathrm{Al}-\mathrm{O}$ bonds decreases by one at the failure point.

$314 \mathrm{C} 3 \mathrm{~A}$ column loses its structural character after one $\mathrm{Al}-\mathrm{O}$ bond 315 is lost, which occurs after the system's failure point at $\sim 11 \%$ 316 elongation.

317 From Figures 7 and 8, it is evident that the change in the 318 number of $\mathrm{Ca}-\mathrm{O}$ bonds is much less than that observed for the $319 z(c)$ direction. Moreover, these changes are mainly due to the $320 \mathrm{Ca}$-water interactions because the conformation of the $\mathrm{C} 3 \mathrm{~A}$ 321 column is not changed during the deformation. The failure 322 mechanism in this case is associated with hydrogen bond 323 breaking between neighboring C3A columns.

324 The monocline structure in ettringite, which causes some 325 shear stress, and hydrogen bond breaking are responsible for 326 the oscillatory nature of Figures 9 and 10 .

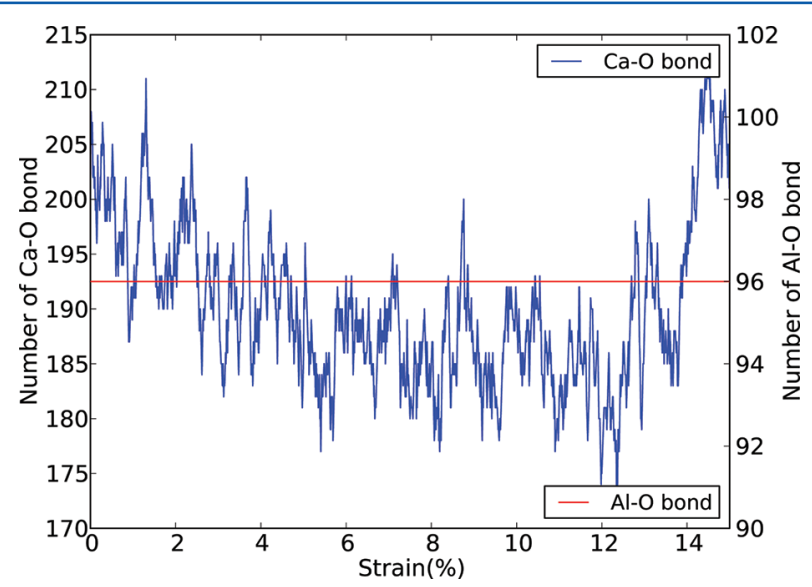

Figure 9. The number of $\mathrm{Ca}-\mathrm{O}$ bonds decreases linearly during compression in the $y$ triclinic direction, and a small increase is observed after failure at $\sim 12 \%$, while the number of $\mathrm{Al}-\mathrm{O}$ bonds remains constant even after the failure point.

\section{SUMMARY AND CONCLUSIONS}

327 We have developed and validated a first-principles-derived 328 ReaxFF reactive force field for ettringite and applied it to study 329 the general mechanical properties and atomistic failure 330 mechanisms of the ettringite mineral. In particular, we 331 determined the structural stability and geometry of ettringite 332 at ambient temperature to be in close agreement with

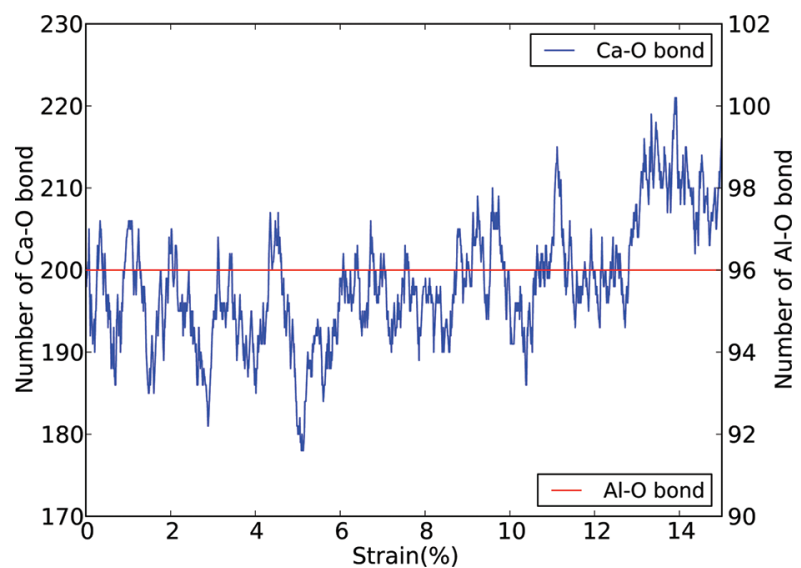

Figure 10. The number of $\mathrm{Ca}-\mathrm{O}$ bonds changes little during tensile deformation in the $y$ triclinic direction, with a slight increase after failure at $\sim 12 \%$.

experimental RDFs, its adiabatic elastic constants to be in 333 quantitative agreement with experimentally obtained constants, 334 and from isothermal-isobaric RMD simulations, the sequence 335 of molecular events that lead to its failure under mechanical 336 compression and tension. We found that $\mathrm{Ca}-\mathrm{O}$ bond breaking 337 in the C3A column leads to failure during compression and 338 tension, while hydrogen bond network formation leads to an 339 increase in plastic strain during compression.

340

The results presented here are essential for understanding 341 the role of ettringite in cement and concrete properties. We are 342 now using this ReaxFF force field to study the hydration 343 kinetics of ettringite and the effect of water on ettringite (and 344 delayed ettringite formation, DEF) as it pertains to the long- 345 term degradation of cement paste and concrete properties. This 346 will be the subject of a subsequent publication.

347

\section{ASSOCIATED CONTENT}

348

S Supporting Information

349

Description of the ReaxFF reactive force field developed. This 350 material is available free of charge via the Internet at http:// 351 pubs.acs.org.

\section{AUTHOR INFORMATION}

*E-mail: ajaramil@caltech.edu (A.J.-B.); wag@wag.caltech.edu 355 (W.A.G.).

Notes

The authors declare no competing financial interest.

\section{ACKNOWLEDGMENTS}

The authors would like to thank Adri van Duin for providing 360 the Al/O ReaxFF parameters and Hegoi Manzano for providing 361 the corresponding $\mathrm{Ca} / \mathrm{O}$ parameters. L.L. performed the work 362 associated with his contributions in this paper during a visiting 363 appointment at the Materials and Process Simulation Center in 364 Caltech. This material is based on work supported by the 365 Department of Transportation, Federal Highway Administra- 366 tion (FHWA), under Award Number BAA No. DTFH61-09-R- 367 00017. Special thanks to Dr. Kunik Lee from FHWA for HIS 368 support of this program. 


\section{$370 \square$ REFERENCES}

371 (1) Lee, H.; Cody, R. D.; Cody, A. M.; Spry, P. G. Environ. Eng. 372 Geosci. 2003, 9, 313-326.

373 (2) Santhanam, M.; Cohen, M. D.; Olek, J. Cem. Concr. Res. 2001, 31, 374 845-851.

375 (3) van Duin, A. C. T.; Dasgupta, S.; Lorant, F.; Goddard, W. A. J. 376 Phys. Chem. A 2001, 105, 9396-9409.

377 (4) Chenoweth, K.; A.C.T., v. D.; P., P.; Cheng, M.; J., O.; W.A., G. I. 378 J. Phys. Chem. C 2008, 112, 14645-14654.

379 (5) van Duin, A. C. T.; Strachan, A.; Stewman, S.; Zhang, Q. S.; Xu, 380 X.; Goddard, W. A. J. Phys. Chem. A 2003, 107, 3803-3811.

381 (6) Zhang, Q.; Cagin, T.; van Duin, A.; Goddard, W. A.; Qi, Y.; 382 Hector, L. G. Phys. Rev. B 2004, 69, 11.

383 (7) Cheung, S.; Deng, W. Q.; van Duin, A. C. T.; Goddard, W. A. J. 384 Phys. Chem. A 2005, 109, 851-859.

385 (8) Chenoweth, K.; Cheung, S.; van Duin, A. C. T.; Goddard, W. A.; 386 Kober, E. M. J. Am. Chem. Soc. 2005, 127, 7192-7202.

387 (9) van Duin, A. C. T.; Zeiri, Y.; Dubnikova, F.; Kosloff, R.; Goddard, 388 W. A. J. Am. Chem. Soc. 2005, 127, 11053-11062.

389 (10) Nielson, K. D.; van Duin, A. C. T.; Oxgaard, J.; Deng, W. Q.; 390 Goddard, W. A. J. Phys. Chem. A 2005, 109, 493-499.

391 (11) Kim, S.; Persson, P.; Kumar, N.; Sofo, J.; Kubicki, J.; van Duin, 392 A. J. Phys. Chem. A 2011, Submitted.

393 (12) Manzano, H.; Buehler, M.; Pellenq, R.; van Duin, A. In 394 preparation for submission.

395 (13) Jaguar; Schrodinger, LLC: New York, 2011.

396 (14) Schultz, P. A.; Muller, P. SeqQuest Electronic Structure Code; 397 Sandia National Laboratories: Albuquerque, NM, 2011.

398 (15) Plimpton, S. J. Comput. Phys. 1995, 117, 1-19.

399 (16) Moore, A. E.; Taylor, H. F. W. Acta Crystallogra., Sect. B 1970, B $40026,386$.

401 (17) Rappe, A. K.; Goddard, W. A. J. Phys. Chem. 1991, 95, 33584023363.

403 (18) Speziale, S.; Jiang, F. M.; Mao, Z.; Monteiro, P. J. M.; Wenk, H. 404 R.; Duffy, T. S.; Schilling, F. R. Cem. Concr. Res. 2008, 38, 885-889. 